Check for updates

Cite this: RSC Adv., 2019, 9, 20174

\title{
The effect of different surface treatment methods on the physical, chemical and biological performances of a PGA scaffold
}

\author{
Yimin Song, ${ }^{a}$ Minghua Ren, ${ }^{b}$ Yadong Wu, DD ${ }^{* c}$ Siyu Li, ${ }^{b}$ Chun Song, ${ }^{b}$ Fang Wang ${ }^{\mathrm{c}}$ \\ and Yudong Huang ${ }^{\mathrm{C}}$
}

In order to improve the adhesion between a PGA scaffold and islet cells, it is necessary to find a suitable method to modify the scaffold. In this study, the PGA scaffold surface was modified by plasma, polylysine coating and plasma combined with polylysine coating (P-P-PGA). The surface adhesion of the modified PGA scaffold was examined, and the stretchability and infiltration of the PGA scaffold were also tested. Then, the PGA scaffold treated under the optimal treatment conditions was selected to coculture with rat islet cells, and the survival activity of the rat islet cells on the untreated PGA scaffold and the P-P-PGA scaffold was examined via the MTT method. Rhodamine staining and DAPI staining were used to detect the number of islet cells adhered to four groups of scaffolds at different culture time points. The PGA-islet graft in the leg muscle of rats was stained with HE to perform the PGA-islet graft pathological examination. The experimental results showed that when the plasma treatment power was $240 \mathrm{~W}$, the processing time was $4 \mathrm{~min}$; the concentration of the polylysine coating solution was $2 \mathrm{mg}$ $\mathrm{ml}^{-1}$, the tensile strength of the PGA scaffold was $320.45 \mathrm{MPa}$ and the amount of infiltration of the PGA scaffold by the serum medium presented the maximum value: $3.17 \mathrm{~g} \mathrm{~g}^{-1}$. The MTT survival activity test results showed that after $3 \mathrm{~d}$ of culture, the survival activity of the islet cells of the treated PGA scaffold culture group $(2.02 \pm 0.13)$ was significantly different from the survival activity of the islet cells of the untreated PGA scaffold culture group $(1.93 \pm 0.10)$. The survival activities of the islet cells in the experimental groups $(1.60 \pm 0.13,1.40 \pm 0.12)$ were still higher than those of the control groups $(0.96 \pm$ $013,0.69 \pm 0.09$ ) at 15 and $21 \mathrm{~d}$. The results of the rhodamine and DAPI staining showed that with the increase in culture time, the number of the adherent cells in each group increased, and the number of the adherent islet cells in the experimental group was higher than that in the untreated group. The HE staining results showed that the islet cells on the P-P-PGA scaffold were more than those on the untreated PGA scaffold. After modification of the PGA scaffold, the adhesion of the islet cells improved, which was conducive to the growth of islet cells. These results confirmed that the plasma combined with polylysine coating treatment could enhance the adhesion of the PGA scaffold surface, so that the scaffold and the islet cells exhibited better adhesion and biocompatibility, and the modified PGA scaffold (P-P-PGA) could be used as a promising islet cell scaffold.

Received 19th March 2019 Accepted 6th June 2019

DOI: $10.1039 / \mathrm{c} 9 \mathrm{ra0} 2100 \mathrm{k}$ rsc.li/rsc-advances the most actively researched biomaterial, ${ }^{\mathbf{5 , 6}}$ which has been approved by the Food and Drug Administration (FDA). ${ }^{7,8}$ Due to its superior biodegradability ${ }^{\mathbf{9 - 1 1}}$ and biocompatibility ${ }^{\mathbf{1 2 - 1 4}}$ and other excellent properties, PGA can be a potential and promising candidate for biological cell scaffolds. ${ }^{15-18}$

Scaffolds with a specific shape and a linked porous structure can be beneficial for the cell adherence and growth, ${ }^{19-21}$ and they have been widely applied in bone repair and bone tissue engineering. ${ }^{22-24}$ In recent years, some researchers have made great efforts to improve the adhesion properties of the PGA scaffold. Some researchers applied a polyurethane coating to the surface of the PGA scaffold materials for modification to promote cell adhesion, growth and proliferation. ${ }^{25}$ Others dissolved biodegradable polymers, such as polyglycolic acid (PGA), 
poly-L-lactic acid (PLLA), and polylactic acid (PLGA), in suitable solvents to prepare nanofiber scaffolds; then, they used the oxygen plasma treatment and hydrophilic acrylic acid (AA) in situ grafted on the surface to modify the surface of the scaffold. Thus, the cell adhesion and proliferation capability improved for co-culture. ${ }^{26}$ Others precoated the polymer scaffold with fibronectin, which is an important protein that can improve the efficiency of cell adhesion. ${ }^{27,28}$ In addition, the plasma treatment is an effective and widely used method for modifying the material surface, ${ }^{29-31}$ and polymer surfaces can be modified by the plasma treatment to improve cell adhesion and promote cell growth. ${ }^{32}$ At present, in order to improve cell adhesion, a variety of surface modification treatment methods have been used in tissue engineering polymer scaffolds. ${ }^{33-35}$

Islet cell transplantation is an ideal treatment for diabetes $^{36-38}$ because the immunogenicity of the graft can be reduced and the immunological rejection of the receptor can be eliminated if the cells are transplanted after in vitro culture. ${ }^{39,40}$ However, it is difficult for the islet cells to survive in an in vitro culture; thus, it is extremely important to select an appropriate scaffold for the islet cell growth. As polylysine exhibits strong adhesion to the surface of the cell membrane, it can promote cell adhesion and growth. ${ }^{41,42}$

In this study, the PGA scaffold was modified by plasma, polylysine coating and plasma combined with the polylysine coating to improve the adhesion between the PGA scaffold and the islet cells. The effects of plasma treatment with polylysine coating and plasma combined with polylysine coating on the PGA scaffold adhesion were studied by stretchability and infiltration tests. Finally, better modification conditions were selected for the surface treatment of the PGA scaffold, making it more suitable for the islet cell adhesion and growth.

\section{Materials and methods}

\subsection{Materials}

PGA scaffolds were obtained from Synthecon, USA. Polylysine was purchased from Sigma, USA. RPMI-1640 medium was supplied by Hyclone, USA. Healthy adult Wistar rats were provided by the experimental animal center of the hospital affiliated to Harbin Medical University. Fetal bovine serum was supplied by Hyclone, USA. Penicillin and streptomycin were purchased from Hyclone, USA. Hank's solution was provided by Gibco, USA. MTT and DMSO were purchased from Amresco, USA. All chemicals were used without further purification.

\subsection{Plasma treatment on PGA scaffold}

PGA scaffolds were cut into $2 \mathrm{~cm} \times 2 \mathrm{~cm}$ samples and placed inside the plasma cavity. The vacuum pump and the high frequency power supply were turned on and tuned to produce air plasma in the plasma cavity. The power and the reaction time were recorded after the completion of the air cooled plasma activation treatment and then, the energetic power and the vacuum pump were turned off. Finally, the scaffold was removed and set aside.

\subsection{Coating treatment on the surface of PGA scaffold}

Prior to the experiment, polylysine mixtures in aqueous solutions at different concentrations $\left(0.5 \mathrm{mg} \mathrm{ml}^{-1}, 1 \mathrm{mg} \mathrm{ml}^{-1}, 2 \mathrm{mg}\right.$ $\mathrm{ml}^{-1}$, and $4 \mathrm{mg} \mathrm{ml}^{-1}$ ) were prepared. The PGA scaffold (sample of $2 \mathrm{~cm} \times 2 \mathrm{~cm}$ ) was then immersed into the above solution for coating treatment, and the soaking time was recorded. After the reaction, the scaffold was dried for $24 \mathrm{~h}$ for backup use.

\subsection{Plasma combined with coating treatment}

After the air-cooled plasma activation, the high-energy power supply and the vacuum pump were turned off; the sample was quickly removed and immersed into a polylysine coating solution of a certain concentration, and the dip time was recorded. After the reaction, the support was removed and the scaffold was air dried for $24 \mathrm{~h}$ for backup use.

\subsection{Stretchability test of PGA scaffold}

The PGA scaffold was cut into $1 \mathrm{~cm} \times 5 \mathrm{~cm}$ pieces and then, it was clamped between the two parallel beams of the CMT8102 electronic universal testing machine (New think again co., LTD., China). The stretchability was tested at the loading speed of $2 \mathrm{~mm} \mathrm{~s}^{-1}$.

\subsection{Infiltration performance test of PGA scaffold}

The PGA scaffold was cut into pieces and immersed into the serum culture medium (RPMI-1640 medium). Then, this mixture was transferred into a pipe, which was $50 \mathrm{~mm}$ in length and $0.35 \mathrm{~mm}$ in diameter. After that, the pipe was placed vertically in a HB-312 type fiber infiltration meter (Chemical Research Institute in Beijing, China) to test the infiltration performance, and the test was automatically terminated when the difference between two measurements was less than $0.1 \mathrm{mg}$.

\subsection{Cell culture}

Healthy adult Wistar rats, weighing 150-200 g, were randomly selected from male and female rats, and this was approved by the animal ethics committee of the hospital affiliated to Harbin Medical University. The rats were isolated to culture the islet cells. Prior to the assay, the scaffolds were sterilized under the ultraviolet light. The experiment was divided into two groups, i.e., the islet cells cultured on the untreated PGA scaffold (control group) and the scaffold treated under the optimal surface modification conditions. RPMI-1640 served as the culture medium; then, $10 \%$ fetal bovine serum (FBS), 1\% penicillin (10 000 units per $\mathrm{ml}$ ) and 1\% streptomycin (10 $000 \mu \mathrm{g}$ $\mathrm{ml}^{-1}$ ) were added. After that, the cells were cultured at $37{ }^{\circ} \mathrm{C}$ under $5 \% \mathrm{CO}_{2}$ in a thermostat incubator (McO-15ac, Sanyo, Japan).

\subsection{MTT assay}

The islet cells of the two culture groups were tested by an MTT assay at $0,3,7,11,15$ and $21 \mathrm{~d}$. The method is described as follows: the islet cells on the scaffold were eluted by Hank's solution, and 10 holes in the first row of the 96-well plate were 
put into the islet cell suspension of the control group (untreated PGA scaffold); each hole was inoculated with $180 \mu \mathrm{L}$ cell suspension ( $\sim 600$ islet cells). The islet cells of the experimental group (scaffold modified by plasma combined with polylysine coating) were inoculated into the second row of 10 holes with the same concentration of the cell suspension. After the cells were inoculated, the 96-well plates were incubated in an incubator with $5 \% \mathrm{CO}_{2}$ at $37^{\circ} \mathrm{C}$ for $24 \mathrm{~h}$. Then, $20 \mu \mathrm{L}$ of MTT with the final concentration of $5 \mathrm{mg} \mathrm{ml}{ }^{-1}$ was added to each hole and cultured at $37{ }^{\circ} \mathrm{C}$ for additional $4 \mathrm{~h}$. After that, the mixture was centrifuged and the supernatant was discarded. Then, $150 \mu \mathrm{L}$ DMSO was added and the suspension was mixed for 5-10 min. Finally, colorimetry was performed using the MK3 type automatic multi-function enzyme marker (Thermo Labsystems, USA), measuring the light absorption value $\left(\mathrm{OD}_{490 \mathrm{~nm}}\right)$ of each hole.

\subsection{Islet cell adhesion experiment}

The experiment was divided into four groups: (1) original PGA scaffold, (2) PGA scaffold treated with plasma, (3) PGA scaffold treated with polylysine, and (4) PGA scaffold treated with plasma combined with polylysine. Ten thousand islet cells were inoculated into the four scaffold groups and incubated in $37^{\circ} \mathrm{C}$ with $5 \% \mathrm{CO}_{2}$ in the incubator. The number of the adherent islet cells after they were cultured for 12, 24 and $48 \mathrm{~h}$ was measured. Then, the islet cells in each group were eluted from the scaffold, and rhodamine and DAPI staining was carried out as follows. The cell culture fluid was discarded and the cells were washed with PBS 3 times (each time for $5 \mathrm{~min}$ ). Then, the cells were fixed by using 4\% PFA (paraformaldehyde) for 15-30 min at room temperature. Next, the cells were washed with PBS 3 times (each time $5 \mathrm{~min}$ ). Then, $0.1 \%$ Triton (Triton $\mathrm{X}-100$ ) was added in order to perforate the cells for $15 \mathrm{~min}$ at room temperature. After that, the cells were washed with PBS 3 times (each time for $5 \mathrm{~min}$ ) and then, they were blocked with $0.5 \%$ BSA at room temperature for $15 \mathrm{~min}$. Rhodamine was then added until the fluid could cover the cells. After incubation for $1 \mathrm{~h}$ in the dark, the cells were washed with PBS 3 times (each time for $5 \mathrm{~min}$ ). Then, DAPI was added and the amount of the fluid should be enough to immerse the cells. After being stained at room temperature for $15 \mathrm{~min}$, the cells were washed for three times with PBS ( 5 min for each time). The observations of islet cells in the four groups were performed using an inverted fluorescence microscope (Olympus, Japan). Five observation points in each group were randomly selected to calculate the mean value of the adhesion degree, and each experimental group was repeated in three holes.

\subsection{In vivo experiment}

The PGA-islet cells were embedded into the leg muscles of the healthy adult Wistar rats. Eleven days after the surgery, the specimens were taken out, embedded in paraffin and sectioned. Then, wood grain eosin (HE) staining was performed, followed by a water rinse and then dehydration with $80 \%, 90 \%, 95 \%$ and $100 \%$ ethanol, respectively. Xylene was used to produce a transparent section and then, the section was sealed with neutral gum. After that, the nuclei were stained with hematoxylin, and the cell plasma and other connective tissues were stained with eosin.

\subsection{Statistical analysis}

The MTT test results of the rat islet cells in different groups were expressed as mean \pm standard deviation (SD) at five time points and were completed by SAS 9.1 software. All quantitative data of the islet cell adhesion test were expressed as mean \pm standard deviation (SD). ANOVA was carried out to compare the differences among four groups. Statistical comparisons were performed using the SPSS 17.0 software. A statistical significance was considered when $p<0.05$.

\section{Results and discussion}

\subsection{Plasma treatment}

3.1.1 Effect of treatment power on the tensile strength of PGA scaffold. The stress-displacement curves of PGA treated with different powers are shown in Fig. 1. The initial strength of the scaffold is about 205.69 MPa. With the increase in the plasma treatment power, the tensile strength of PGA increased first and then decreased. When the treatment power was $120 \mathrm{~W}$, the oxidation of plasma treatment played the main role, and the etching effect just appeared. Therefore, the tensile strength of the PGA material exhibits negligible change compared to that of the untreated samples, which is about 164.27 MPa. With the increase in the plasma processing power, the etching ability of plasma becomes stronger gradually. When the treatment power was $240 \mathrm{~W}$, the etching effect was the strongest, and the tensile strength reached the maximum value of about 299.78 MPa. This was because moderate etching could remove impurities and eliminate the defects, stress concentration points and a weak boundary layer on the surface of the PGA scaffolds. Therefore, moderate etching could properly improve the strength of the PGA scaffolds. When the treatment power was $360 \mathrm{~W}$, the etching effect continued to increase, and the tensile strength

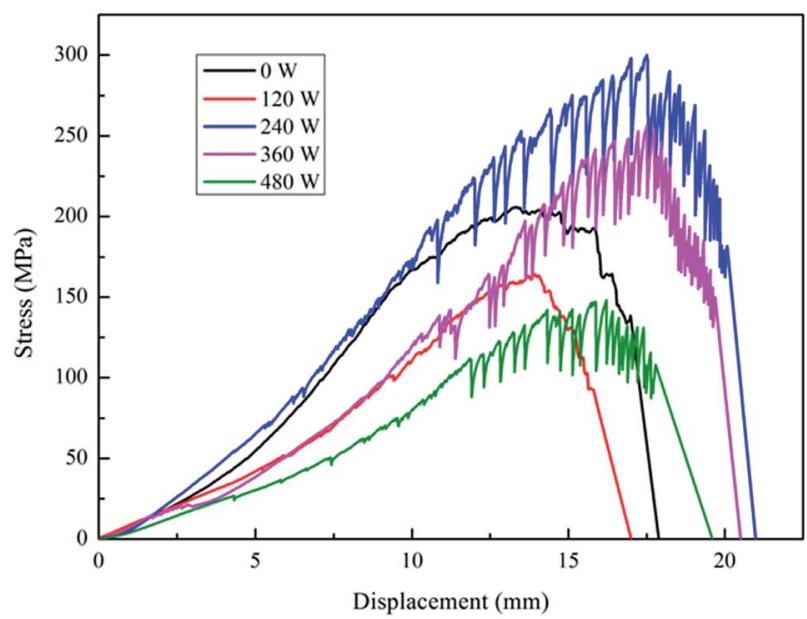

Fig. 1 Effect of plasma power on the mechanical performance of PGA scaffold. 
was approximately $256.46 \mathrm{MPa}$. The etching effect was relatively moderate when the plasma treatment powers were $240 \mathrm{~W}$ and $360 \mathrm{~W}$. Therefore, the tensile properties of the PGA scaffolds improved when the plasma treatment powers were $240 \mathrm{~W}$ and $360 \mathrm{~W}$. When the plasma treatment power reached $480 \mathrm{~W}$, the tensile strength of PGA decreased to $148.11 \mathrm{MPa}$. At this high power, excessive etching caused the diameter of the fiber to become smaller, and the amount of the surface stress concentration points increased. Thus, the tensile strength of the PGA scaffold treated with $480 \mathrm{~W}$ plasma decreased significantly, as shown in Fig. 1. The stress-displacement curves of PGA scaffolds treated with different powers show that the tensile strength of the PGA scaffolds is the best when the treatment power is $240 \mathrm{~W}$.

The stress-displacement curves (Fig. 1) show a large fluctuation of the force value at the maximum point. This may be caused by the loosening of the plasma-treated PGA materials, which could be found by the naked eye observation of the treated PGA scaffolds. When the treatment power was $120 \mathrm{~W}$, the PGA scaffold is not very loose, and a continuous fracture can be basically guaranteed when a fracture occurs. Therefore, there is no substantial swing phenomenon of the force value, which is similar to that of the untreated samples. When the treatment power continued to increase, the degree of loosening of the PGA scaffold increased. Because the tested material is made of intertwined PGA scaffolds, when the material became loose, the time difference between the initial fracture and the next resulted in an up and down swing of the force value. The displacement of the samples treated with $240 \mathrm{~W}, 360 \mathrm{~W}$ and $480 \mathrm{~W}$ plasma powers could also be explained in the same way. The displacements of the samples treated with the plasma powers of $240 \mathrm{~W}, 360 \mathrm{~W}$ and $480 \mathrm{~W}$ were larger than that of the untreated and the $120 \mathrm{~W}$ samples.

3.1.2 Effect of treatment power on the infiltration of PGA scaffold. Fig. 2 depicts the infiltration curve between the PGA scaffold and the serum culture medium after the plasma treatment with different powers. The serum culture medium is

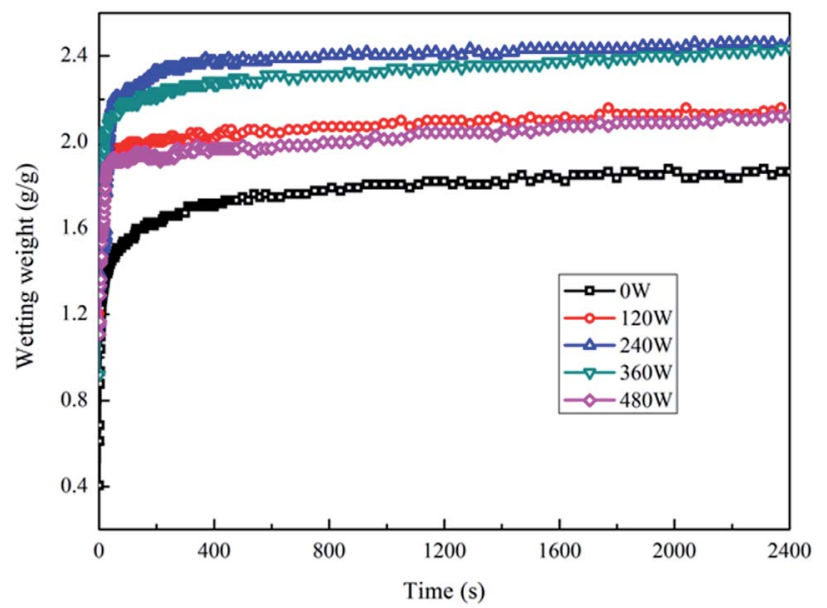

Fig. 2 Effect of treatment power on the wettability of PGA scaffold and serum. the nutritional component of cells; thus, impregnating PGA scaffold with the serum-containing cell culture medium could improve the infiltration performance of the PGA scaffold. Only when the scaffold contained a large amount of the serum culture medium, it could provide sufficient nutrients and a framework for cell attachment. Therefore, the infiltration of the plasma-treated PGA scaffolds by the serum culture media was tested to evaluate the effect of plasma power. As seen in Fig. 2, the infiltration of the treated PGA scaffolds by the serum culture media is greater than that of the untreated sample by the serum culture media. With the increase in the plasma power, the infiltration increased first and then decreased. When the power of the plasma treatment was $240 \mathrm{~W}$, the infiltration of the PGA scaffold by the serum culture medium reached its maximum. This is mainly due to the fact that the number of the oxygen-containing functional groups on the surface of the plasma-treated PGA scaffold is the largest when the plasma treatment power is $240 \mathrm{~W}$. Water is a polar solvent, and a serum culture medium contains plenty of water. When the number of polar functional groups on the surface of PGA scaffolds increases significantly, according to the principle of similarity compatibility, the PGA scaffolds with more polar functional groups are more compatible with polar solvents. Therefore, at the plasma treatment power of $240 \mathrm{~W}$, the content of the oxygen-containing functional groups on the surface of the PGA scaffolds was the largest and polarity was the strongest. Therefore, the infiltration of the PGA scaffolds by the serum culture medium is the best with the infiltration amount reaching $2.46 \mathrm{~g} \mathrm{~g}^{-1}$. Through the above research, it can be concluded that the optimum power of the plasma treatment is $240 \mathrm{~W}$.

3.1.3 Effect of plasma treatment time on the tensile strength of PGA scaffold. The tensile strength of the plasmatreated PGA scaffolds with different times is presented in Fig. 3. It can be seen that the tensile strength of the PGA scaffolds increases first and then decreases with the prolongation of the plasma treatment time. When the treatment time is $4 \mathrm{~min}$,

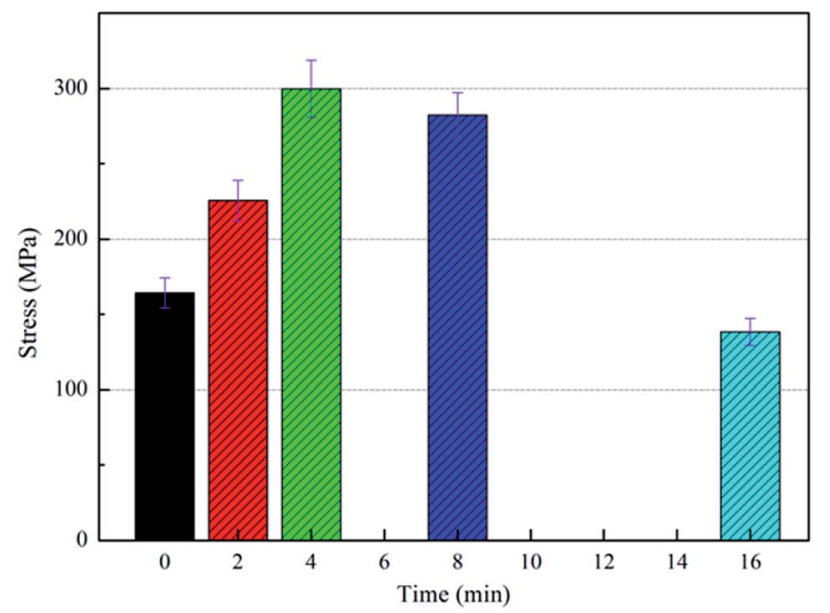

Fig. 3 Effect of plasma treatment time on the mechanical performance of PGA scaffolds. 
the tensile strength of PGA scaffolds reaches the maximum value of approximately 299.78 $\mathrm{MPa}$. A short treatment time has negligible effect on the surface of the PGA scaffold; thus, the tensile strength of the scaffold is similar to that of the untreated scaffold when the treatment time is $2 \mathrm{~min}$. After prolonging the treatment time, the time for particles to wash the surface of PGA scaffolds is also prolonged; thus, the etching effect gradually starts to appear. As mentioned above, moderate etching can remove impurities and eliminate partial defects, stress concentration points and a weak boundary layer on the surface of the PGA scaffold, properly improving the strength of the PGA scaffold. From Fig. 3, it can be observed that the tensile strengths of the PGA scaffolds are 299.78 MPa and 282.18 MPa, corresponding to the scaffolds under the plasma treatment times of $4 \mathrm{~min}$ and $8 \mathrm{~min}$, which lead to the proper etching of the PGA scaffold surface; thus, the tensile property of the PGA scaffolds is better than that of the untreated one. When the plasma treatment time reached $16 \mathrm{~min}$, the tensile strength of the PGA scaffolds decreased significantly to $138.22 \mathrm{MPa}$, which was attributed to the excessive etching that might damage the PGA scaffold, causing thinning of the fibers and increase in surface stress concentration points.

3.1.4 Effect of plasma treatment time on the infiltration of PGA scaffold. The same rule can be illustrated by Fig. 4, which is assigned to the influence of the plasma treatment time on the infiltration of the scaffold. The infiltration of the treated PGA scaffolds by the serum culture medium is higher than that of the untreated group. Moreover, with the increasing plasma treatment time, the infiltration of the PGA scaffolds by the serum culture medium increased first and then decreased. The maximum infiltration of the PGA scaffold by the serum culture medium was $2.46 \mathrm{~g} \mathrm{~g}^{-1}$ when the treatment time was $4 \mathrm{~min}$. This was mainly due to the fact that the amount of oxygencontaining functional groups on the surface of the plasmatreated PGA scaffolds was the largest at the plasma treatment time of $4 \mathrm{~min}$.

The above conclusions are basically the same as that of the infiltration of the PGA scaffolds by the serum culture medium

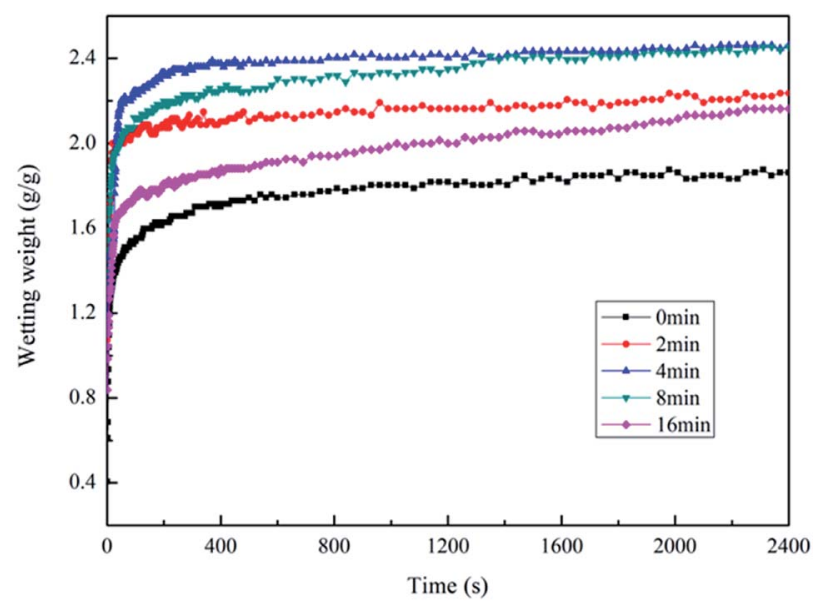

Fig. 4 Effect of plasma treatment time on the wettability of PGA scaffold and serum. treated with different powers. However, the difference is that the infiltration of the PGA scaffolds by the serum culture medium treated with plasma for different times showed no obvious decreasing trend. This may be due to the fact that the energy of the particles is basically the same under a fixed power, but the difference is only the contact times between the particles and the PGA scaffold. A long low-energy particle treatment is not equivalent to the short high-energy particle treatment. Therefore, when the treatment power was determined, the prolongation of the treatment time of the low-energy particles produced less effect than the short treatment of the high-energy particles on the surface of the fibers; thus, the decreasing trend of infiltration of the plasma-treated PGA scaffolds by the serum culture medium was not particularly obvious. After the above research, the optimum technological parameters of the plasma treatment of PGA scaffolds could be determined as follows: the treating power was $240 \mathrm{~W}$, and the treating time was $4 \mathrm{~min}$.

\subsection{Surface coating treatment of PGA scaffold}

3.2.1 Influence of polylysine coating on the tensile strength of PGA scaffold. The tensile strength of PGA scaffolds treated with different concentrations of polylysine coating is displayed in Fig. 5. With the increase in the concentration of the polylysine coating solution, the tensile strength of the PGA scaffold increased slowly at first and then decreased slowly. When the concentration of the polylysine coating solution was $2 \mathrm{mg} \mathrm{ml}^{-1}$, the tensile strength of the PGA scaffolds was the highest, reaching 282.62 $\mathrm{MPa}$. The tensile strengths of the PGA scaffolds treated with other concentrations of polylysine coatings also significantly increased compared with that of the unprocessed PGA scaffold. For example, when the concentration of the polylysine coating solution increased from $0.5 \mathrm{mg} \mathrm{ml}^{-1}, 1 \mathrm{mg}$ $\mathrm{ml}^{-1}$ to $4 \mathrm{mg} \mathrm{ml}^{-1}$, the tensile strength of the PGA scaffolds varied from approximately $215.23 \mathrm{Mpa}, 236.66 \mathrm{Mpa}$ to $210.80 \mathrm{MPa}$, respectively.

This observation could be explained by the moderate hardness of the polylysine coating and the strong bonding force to

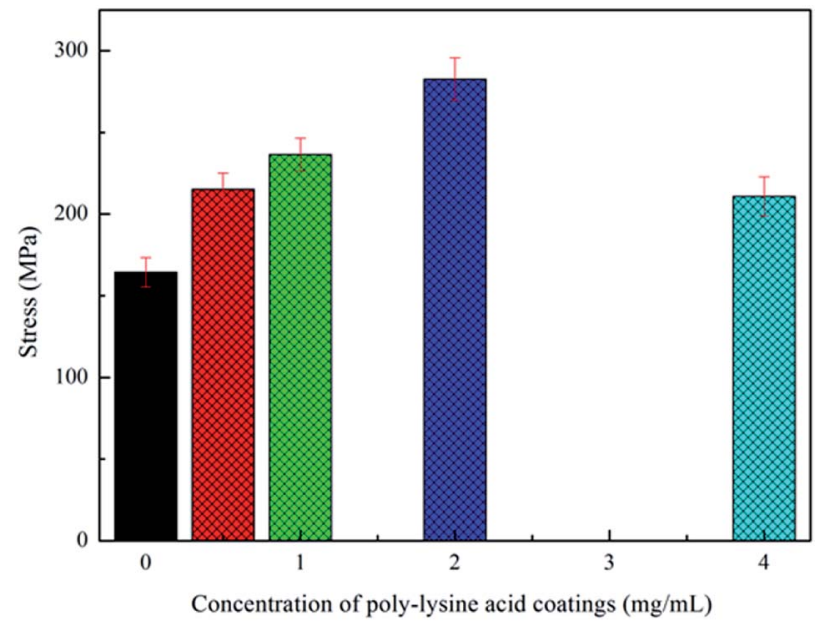

Fig. 5 Influence of polylysine coating solution concentration on the mechanical performance of PGA scaffold. 
the PGA scaffold. Although some of the entangled fibers were separated by the introduction of the coating, the coated fibers were still bonded together by the coating. In addition, there were winding points bonded by the coating, which were also very important to enhance the tensile strength of the PGA scaffolds. Therefore, through the analysis of the tensile strength, it could be confirmed that the polylysine coatings with moderate hardness and viscosity bind the PGA scaffolds formed by filament winding, significantly improving the tensile strength of the PGA scaffolds. However, the polylysine solution exhibited poor fluidity when the concentration of the coating solution was very high, so that it was not easy to form a uniform coating on the surface of the fiber. Therefore, the tensile strength of the scaffold became worse, and the high concentration of polylysine also worsened the flexibility of the scaffold.

3.2.2 Influence of polylysine coating on the infiltration of PGA scaffold. The infiltration curves of PGA scaffolds and serum cultures treated with polylysine coatings at different concentrations are presented in Fig. 6. The infiltration of PGA scaffolds after the application of the polylysine coatings significantly improved. With the increase in the polylysine coating concentration, the maximum infiltration of the scaffolds basically presented a gradual upward trend. Polylysine has a strong ability to absorb water. When it was coated onto the PGA scaffold, the polylysine-coated PGA scaffold displayed good hydrophilicity, so that the scaffold exhibited superior infiltration by the serum culture medium. Based on the relationship between the concentration of the coating solution and the strength of scaffolds, the optimal concentration of the polylysine coating solution could be decided to be $2 \mathrm{mg} \mathrm{ml}^{-1}$.

\subsection{Plasma combined with polylysine surface coating treatment}

The effects of the plasma treatment and the polylysine coating treatment on the performance of PGA scaffolds were studied, and the appropriate process parameters were selected. These treatments could improve the tensile strength of the PGA

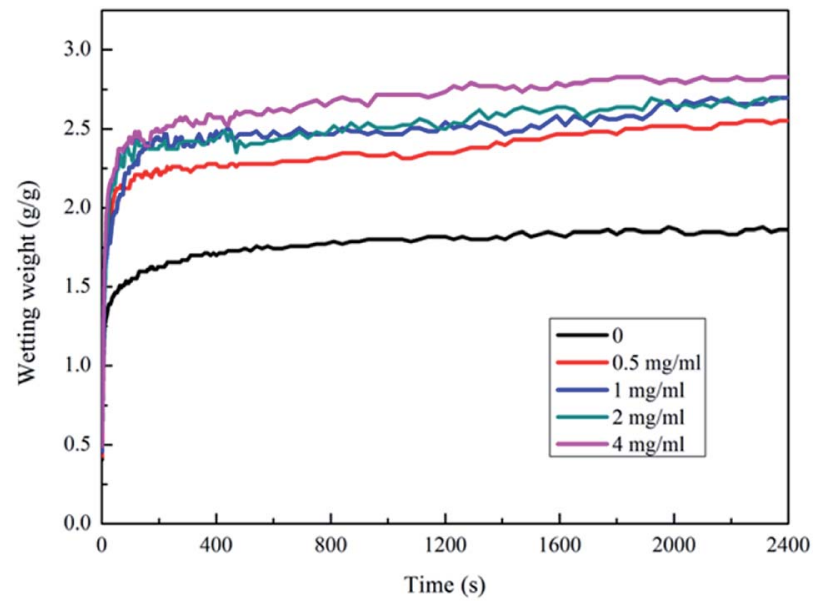

Fig. 6 Wettability of PGA scaffold treated by different concentration polylysine coatings and serum. scaffolds and the infiltration of the PGA scaffolds. Therefore, we combined the two methods to achieve the best treatment effect. We used the plasma power of $240 \mathrm{~W}$ to treat the scaffold for $4 \mathrm{~min}$ and the polylysine coating concentration of $2 \mathrm{mg} \mathrm{ml}^{-1}$ to modify the PGA scaffold. The stress-displacement curve of the PGA scaffolds treated with $240 \mathrm{~W}$ combined with $2 \mathrm{mg} \mathrm{ml}^{-1}$ polylysine coating shows that the tensile strength of the PGA scaffolds reaches $320.45 \mathrm{MPa}$, which is higher than the tensile strengths of the PGA scaffolds treated with plasma alone (299.78 $\mathrm{MPa})$ and the coating alone $(282.62 \mathrm{MPa})$. This suggests that plasma combined with the polylysine coating can greatly improve the tensile strength of the PGA scaffolds.

At the same time, it can be observed in Fig. 7a that the fracture of the PGA scaffold after plasma bonding with the polylysine coating is basically a ductile fracture, and the tensile displacement is also very long. This implies that the polylysine coating makes a strong interfacial bond with the PGA scaffold. Therefore, the mechanical performance of the PGA scaffolds treated by plasma combined with the polylysine coating meets the expected requirements.

The infiltration curve of the plasma combined with polylysine coating PGA scaffold is exhibited in Fig. 7b. The maximum infiltration of the PGA scaffold by the serum culture medium is $3.17 \mathrm{~g} \mathrm{~g}^{-1}$, which is significantly higher than that of the PGA scaffold treated with plasma alone or the polylysine coating alone. This indicates that plasma combined with polylysine coating is useful to improve the infiltration of the PGA scaffold.

Many polar functional groups emerged on the surface of PGA after the plasma treatment, and these groups could improve the surface energy of the PGA scaffolds, so that the tensile strength and wettability of the scaffolds could be improved. At the same time, these polar functional groups could also interact with the polylysine coatings that infiltrated the PGA scaffolds in various physical and chemical ways, thus improving the interfacial bonding between the PGA scaffolds and the polylysine coatings and the infiltration of the PGA scaffolds. Besides, the polylysine coatings could increase the tensile strength of the PGA scaffolds. Furthermore, the coatings exhibited excellent adhesion to and infiltration of the PGA scaffolds, which helped improve the wettability of the polylysine coatings in the serum culture medium.

The application of the plasma treatment combined with the polylysine coating on PGA scaffolds significantly improved the mechanical performance and wettability. The experimental results reveal that the plasma treatment combined with the polylysine coating treatment is an ideal method for the modification of PGA, which can provide an adequate scaffold for cell adhesion and growth in vitro.

\subsection{Analysis of MTT detection results}

As mentioned above, the optimal PGA scaffold modified by plasma combined with polylysine coating was selected to carry out the subsequent analysis. The MTT assay is a method for detecting cell activity and growth. Succinic acid dehydrogenase in the mitochondria of the living cells can reduce exogenous 

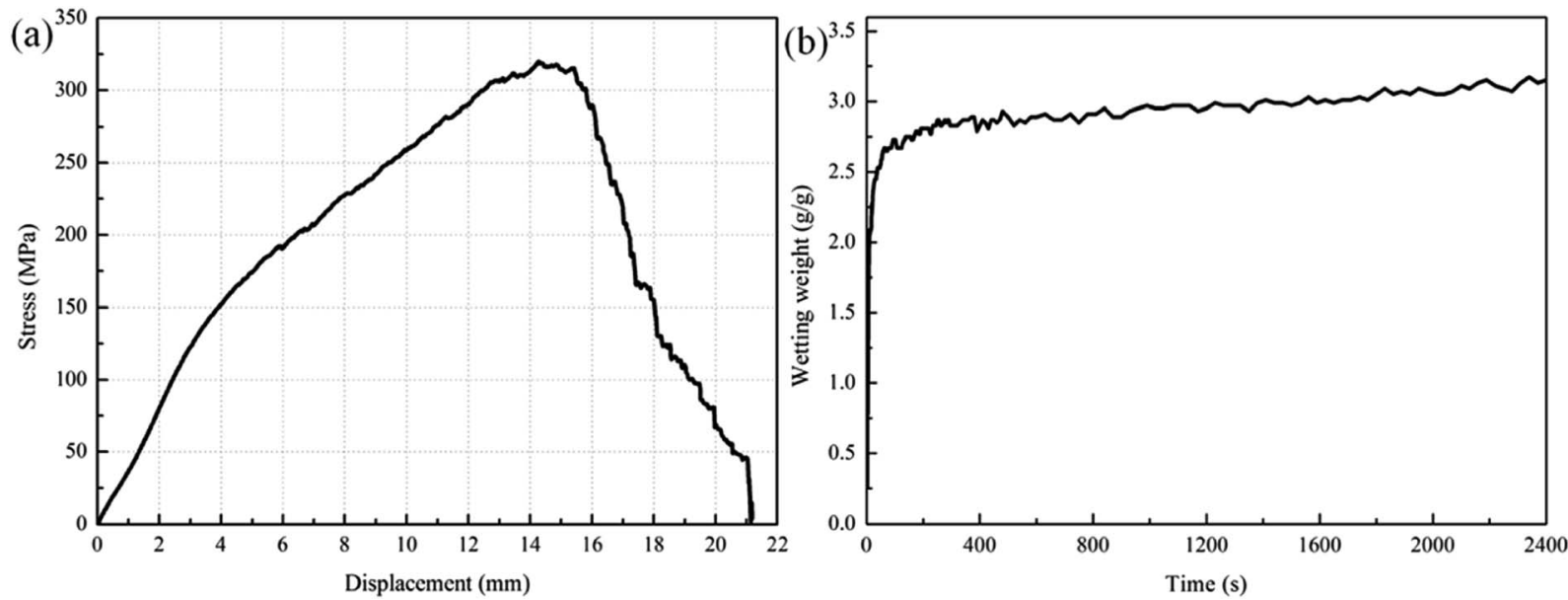

Fig. 7 Influence of plasma treatment combined with polylysine surface coating treatment on (a) the mechanical performance and (b) wettability of PGA scaffold.

MTT to insoluble blue-purple crystals (formazan), which will deposit in cells. However, dead cells cannot exhibit this feature. Thus, we could use DMSO to dissolve the purple crystals and then measure $\mathrm{OD}_{490 \mathrm{~nm}}$ to indirectly determine the number of living cells. Within a certain range of cells, the amount of MTT crystals formed is proportional to the number of cells. Islet cells were non-renewable in this study; thus, the MTT method could be used to detect the survival activity of the islet cells in rats, so that the biological adhesion between the islet cells and the PGA scaffold material could be determined. As the PGA scaffold was modified by plasma combined with the polylysine coating, improving its adhesion performance, the islet cells could adhere to the scaffold and grow. The more the islet cells adhering to the scaffold, the higher the survival activity of islet cells, indicating better biocompatibility between the islet cells and the scaffold.

As displayed in Fig. 8, the islet cells in the control group (untreated PGA scaffold) and the experimental group (PGA

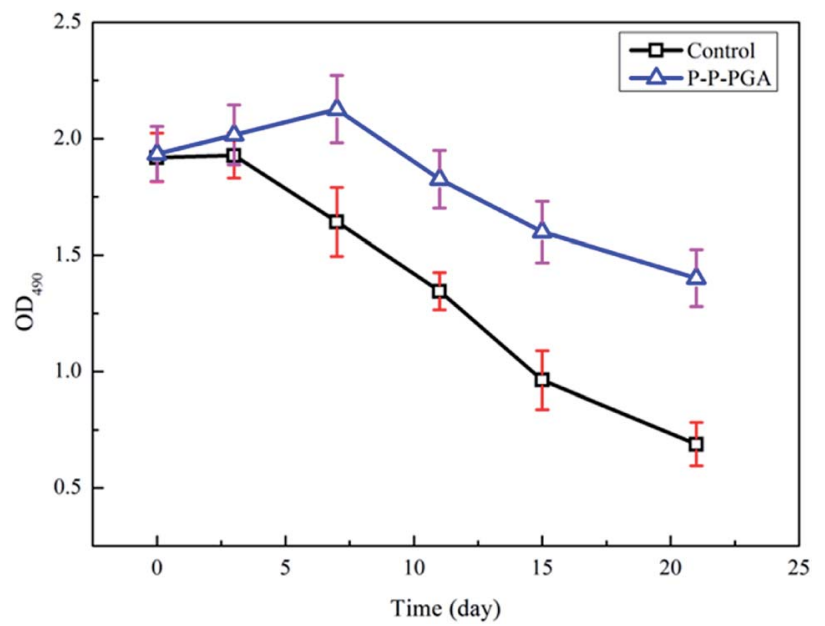

Fig. 8 MTT assay results for PGA scaffolds before (control) and after $(\mathrm{P}-\mathrm{P}-\mathrm{PGA})$ the modification. scaffold modified by plasma combined with polylysine coating, P-P-PGA) at different times determined by the MTT test showed significant differences $(p<0.05)$. The survival activity of the islet cells in the experimental group after $3 \mathrm{~d}(2.02 \pm 0.13)$ was significantly higher than that in the control group $(1.93 \pm 0.10)$. After 15 and $21 \mathrm{~d}$, the islet cell survival activities in the experimental groups $(1.60 \pm 0.13,1.40 \pm 0.12)$ were still higher than those in the control groups $(0.96 \pm 0.13,0.69 \pm 0.09)$. The MTT curve of the control group exhibited a rapid declining stage, which indicated that the survival activity of islet cells decreased obviously. However, the decrease in the MTT curve of the experimental group was slow, implying that the survival activity of the cells slowly decreased, which was obviously higher than that of the control group. It is worth noting that the islet cell survival activity on the scaffold modified by plasma combined with the polylysine coating was significantly higher than that of the cells on the untreated PGA scaffold.

\subsection{Experimental results of islet cell adhesion}

Rhodamine and DAPI staining was used to detect the number of the islet cells adhered to the scaffolds of the 4 groups at different culture times. DAPI stains the nuclei blue and rhodamine stains the cytoskeleton red. The islet cells are classified into single islet cells (represented by a) and a larger islet cell mass (represented by $b$ ) in Fig. 9 .

The adhesion of the islet cells on the scaffolds in 4 groups (1: original PGA scaffold, 2: plasma-treated PGA scaffold, 3: polylysine-coated PGA scaffold, and 4: P-P-PGA) at different culture times is shown in Fig. 10. After being cultured for $12 \mathrm{~h}$, islet cell adhesion numbers were $154 \pm 6.25$ (original PGA scaffold), $191 \pm 8.08$ (plasma-treated PGA scaffold), $243.67 \pm$ 6.12 (polylysine-coated PGA scaffold), and $282.67 \pm 6.09$ (P-PPGA). The numbers of the adhesive islet cells in the experimental group were greater than that in the untreated group, and the difference was statistically significant $(p<0.05)$. When cultured for $24 \mathrm{~h}$, the numbers of the adhered islet cells in the four groups were $169.67 \pm 11.59$ (original PGA scaffold), $343.3 \pm$ 

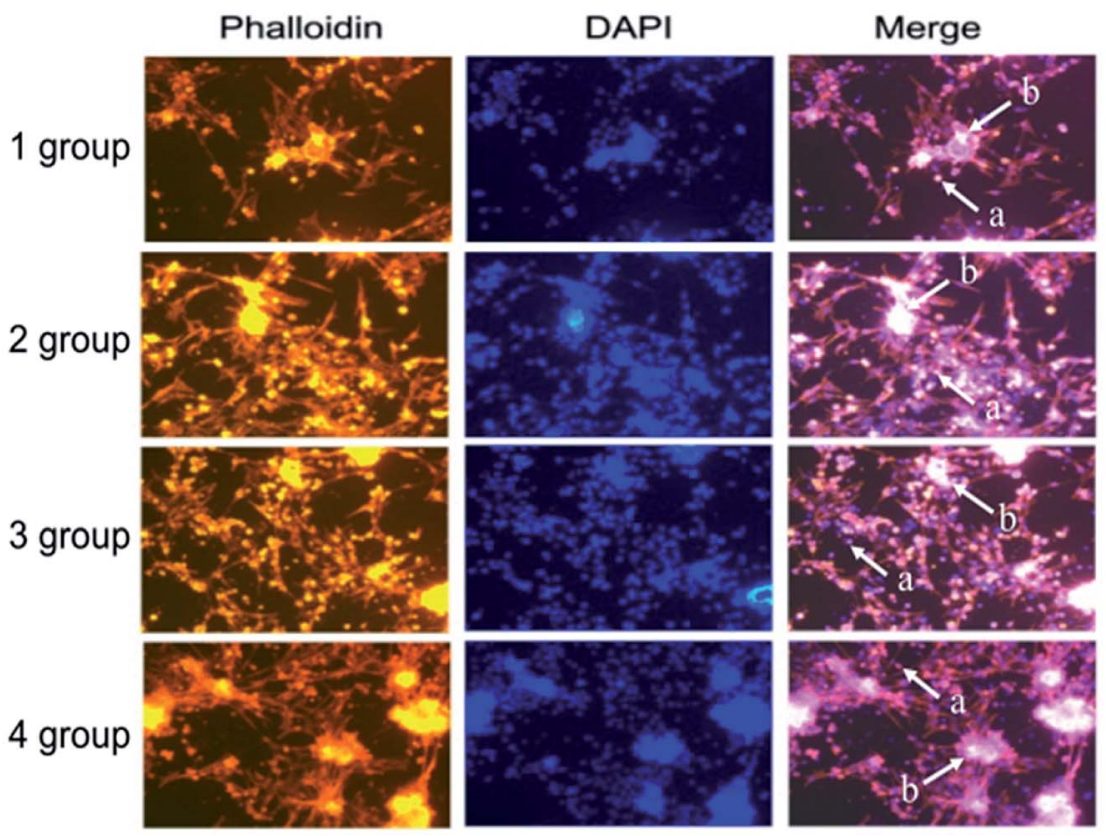

Fig. 9 Images of the stained islet cells in different experimental groups (a represents individual islet cells and b is the larger islet cell mass).

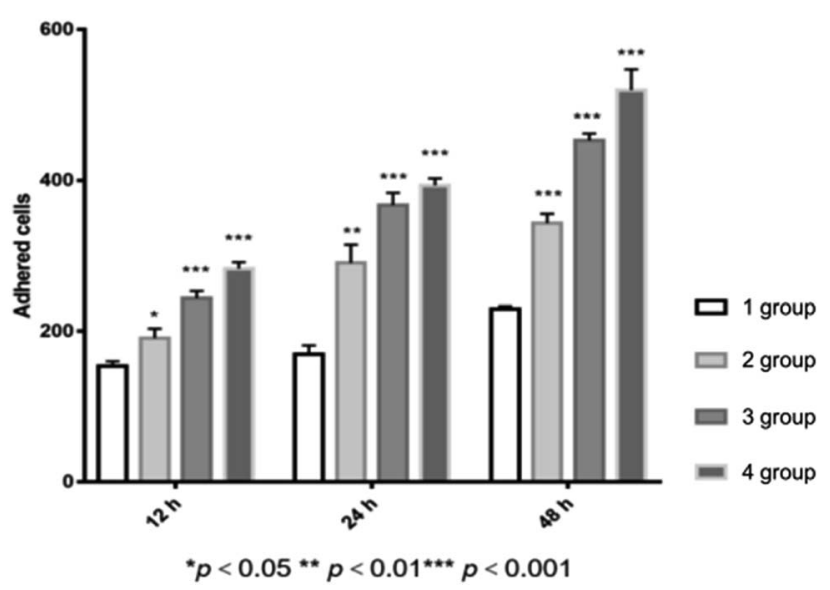

Fig. 10 The adhesion study of islet cells on different PGA scaffolds for $12 \mathrm{~h}, 24 \mathrm{~h}$ and $48 \mathrm{~h}$, respectively $(* p<0.05, * * p<0.01, * * * p<0.001)$

7.41 (plasma-treated PGA scaffold), $453 \pm 5.67$ (polylysinecoated PGA scaffold), and $519.3 \pm 16.11$ (P-P-PGA). As the time increased, the number of the adhered islet cells in each group increased, and the number of the adhered islet cells in the experimental group was higher than that in the untreated group. The number of the adhered islet cells in the fourth group was the highest, and the difference was statistically significant $(p<0.01)$. After being cultured for $48 \mathrm{~h}$, the numbers of the adhered islet cells in the four groups were $229.67 \pm 11.59$ (original PGA scaffold), $343.3 \pm 7.41$ (plasma-treated PGA scaffold), $453 \pm 5.67$ (polylysine-coated PGA scaffold), and $519.3 \pm$ 16.11 (P-P-PGA). The adhesion number of the islet cells on the polylysine-coated PGA scaffold was higher than that on the plasma-treated PGA scaffold. The number on P-P-PGA was still the highest, and the numbers of the islet cells on the three modified scaffolds were significantly higher than that on the untreated scaffold $(p<0.001)$.

Thus, it could be concluded that the plasma treatment or the polylysine coating is able to enhance the adhesion of the PGA scaffold to the islet cells. However, the two methods exhibit different actions and mechanisms to enhance adhesion. The plasma treatment could increase the number of the oxygencontaining groups on the surface of the scaffold, and the polylysine coating exhibited strong adhesion to the surface of the cell membrane. Besides, the enhancement in the adhesion of the PGA scaffold brought by the polylysine coating was more obvious than that caused by the plasma treatment. For the P-PPGA scaffold, we combined the two methods to modify the scaffold, i.e., we applied two individual methods to modify one PGA scaffold in sequence. The results indicated that the P-PPGA scaffold exhibited optimal adhesion. Thus, we can say that the link between the modification methods, from plasma, polylysine coating to plasma combined with the polylysine coating, is a progressive relationship.

\subsection{Pathology results}

The HE staining images of the PGA-islet cell graft embedded in the rat leg muscle were obtained using a light microscope (LM). Fig. 11A shows the PGA scaffold without modification, and Fig. 11B exhibits the PGA scaffold modified by plasma combined with the polylysine coating. In the pictures, (a) is the tubular PGA scaffold, (b) corresponds to the purple dark islet cells (islet cells form round, irregular shapes or cells clumps), (c) corresponds to the lilac striated muscle fiber cells, (d) is fat, and (e) corresponds to the capillaries. The pathological results showed that the islet cells on the PGA-islet graft were in good 


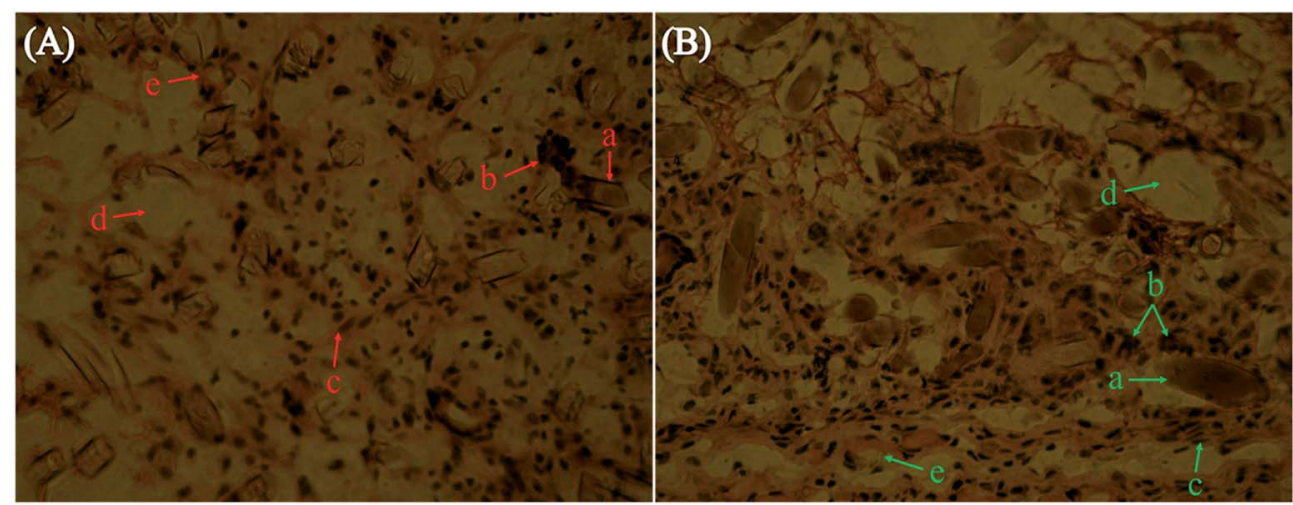

Fig. 11 LM images of PGA-islet cells (A) before and (B) after modification.

shape with close adhesion on the PGA scaffolds. The PGA scaffold simulates the extracellular matrix for supporting the islet cells, making the cells form a three-dimensional growth pattern and keeping some space between the pancreatic islet cells. This can protect the islet cells from the backlog of the muscle fibers, which contain abundant capillaries. Thus, the PGA scaffold-islet grafts could absorb sufficient nutrients to maintain the growth of PGA scaffold-islet grafts in the muscle tissues. Inflammatory cells could not be observed from the pathological results, indicating that the PGA scaffold and muscle fiber tissues have good biocompatibility.

As shown in Fig. 11B, many islet cells adhere and grow around the PGA scaffold modified by plasma combined with polylysine coating. However, the islet cells in the PGA scaffoldislet cell graft in Fig. 11A are not as abundant as those seen in Fig. 11B. The results indicate that cell adhesion on the modified PGA scaffolds is better than that of the untreated PGA scaffolds. This is because the adhesion of the PGA scaffold is improved after the modification by plasma combined with polylysine, which is beneficial to the islet cell adhesion and can create a favorable environment for the islet cells within the muscle tissue.

In recent years, with the continuous development of tissue engineering, the macromolecular biomaterials that can be used as cell scaffolds have been widely used..$^{15,43,44}$ Cell scaffolds can support cell infiltration and protect the cells, ${ }^{45,46}$ and they can also provide a three-dimensional structure for the cell culture in vitro. ${ }^{47}$ In this study, the PGA scaffold was treated with three different surface modification methods, and the optimal conditions for the modification were $240 \mathrm{~W}$ plasma for $4 \mathrm{~min}$ and the polylysine impregnation concentration of $2 \mathrm{mg} \mathrm{ml}^{-1}$. After modification, the adhesion performance of the PGA scaffold could be increased. Besides, it could increase the ingrowth access and the surface adhesion area for the islet cells; thus, the PGA scaffold provides an excellent condition for the adhesion of islet cells.

\section{Conclusion}

This study used three methods, namely, plasma, polylysine coating and plasma combined with polylysine coating to modify
PGA scaffolds. The results confirmed that plasma combined with the polylysine coating is the best treatment method for the modification of the PGA scaffold. When the plasma processing power was $240 \mathrm{~W}$, the treatment time was $4 \mathrm{~min}$, and the polylysine coating liquid concentration was $2 \mathrm{mg} \mathrm{ml}^{-1}$, the tensile strength of the PGA scaffold was $320.45 \mathrm{MPa}$ and the infiltration amount of the scaffold with the serum culture was up to $3.17 \mathrm{~g} \mathrm{~g}^{-1}$. The PGA scaffold treated under the optimal conditions was co-cultured with islet cells. The results of the MTT test indicated that the survival activity of the islet cells in the modified scaffold was higher than that of the untreated scaffold. Rhodamine with DAPI staining results showed that with the increase in the incubation time, the number of the adhesive cells increased in each group, and it was higher in the experimental group than in the untreated group. However, the number of the islet cells adhered in the plasma-conjugated polylysine treatment (P-P-PGA) group was the highest among the four groups, and the difference was statistically significant $(p<0.01)$. The HE staining results also proved that the adhesion of the modified scaffold was better than that of the original scaffold. Therefore, it can be concluded that the PGA scaffold modified by plasma combined with the polylysine coating can be potentially used as a scaffold for islet cells.

\section{Statement}

All animal procedures were performed in accordance with the Guidelines for Care and Use of Laboratory Animals of the US National Institutes of Health (NIH) and approved by the Animal Ethics Committee of Harbin Medical University.

\section{Conflicts of interest}

There are no conflicts to declare.

\section{Acknowledgements}

This study was supported by a grant from the National Natural Science Foundation of China (No. 30570931). This study was also financially supported by China Postdoctoral Science Foundation (2013M541372). We would like to acknowledge the 
financial support from Heilongjiang Postdoctoral Fund ( $\mathrm{LBH}-$ Z13086). This study was also supported by the Fundamental Research Funds for the Central Universities (Grant No. HIT. NSRIF. 2015047).

\section{References}

1 L. E. Niklason, J. Gao, W. M. Abbott, K. K. Hirschi, S. Houser, R. Marini and R. Langer, Functional arteries grown in vitro, Science, 1999, 284, 489-493.

2 H. F. Liu, X. M. Li, X. F. Niu, G. Zhou, P. Li and Y. B. Fan, Improved hemocompatibility and endothelialization of vascular grafts by covalent immobilization of sulfated silk fibroin on poly(lactic-co-glycolicacid) scaffold, Biomacromolecules, 2011, 12, 2914-2924.

3 K. A. Athanasiou, G. G. Niederauer and C. M. Agrawal, Sterilization, toxicity, biocompatibility and clinical applications of polylactic acid polyglycolic acid copolymers, Biomaterials, 1996, 17, 93-102.

4 J. Nikolovski and D. J. Mooney, Smooth muscle cell adhesion to tissue engineering scaffolds, Biomaterials, 2000, 21, 20252032.

$5 \mathrm{H}$. Cao and N. Kuboyama, A biodegradable porous composite scaffold PGA/beta-TCP for bone tissue engineering, Bone, 2010, 46, 386-395.

6 Y. C. Wang, M. C. Lin, D. M. Wang and H. J. Hsieh, Fabrication of a novel porous PGA-chitosan hybrid matrix for tissue engineering, Biomaterials, 2003, 24, 1047-1057.

7 J. P. Jarow, H. U. Ahmed, P. L. Choyke, S. S. Taneja and P. T. Scardino, Partial gland ablation for prostate cancer: Report of a Food and Drug Administration, American Urological Association, and Society of Urologic Oncology Public Workshop, Urology, 2016, 88, 8-13.

8 M. F. Meek and J. H. Coert, US Food and Drug Administration/Conformit Europe-approved absorbable nerve conduits for clinical repair of peripheral and cranial nerves, Ann. Plast. Surg., 2008, 60, 466-472.

9 L. Yu, Z. Zhang, H. Zhang and J. Ding, Biodegradability and biocompatibility of thermoreversible hydrogels formed from mixing a sol and a precipitate of block copolymers in water, Biomacromolecules, 2010, 11, 2169-2178.

10 A. P. Acharya, J. S. Lewis and B. G. Keselowsky, Combinatorial co-encapsulation of hydrophobic molecules in poly(lactide-co-glycolide) microparticles, Biomaterials, 2013, 34, 3422-3430.

11 I. B. Bajaj and R. S. Singhal, Enhanced production of $\operatorname{poly}(\gamma-$ glutamic acid) from bacillus licheniformis NCIM2324 by using metabolic precursors, Appl. Biochem. Biotechnol., 2009, 159, 133-141.

12 K. G. Battiston, R. S. Labow and J. P. Santerre, Protein Binding Mediation of Biomaterial-Dependent Monocyte Activation on a Degradable Polar Hydrophobic Ionic Polyurethane, Biomaterials, 2012, 33, 8316-8328.

13 R. Langer and J. P. Vacanti, Tissue engineering, Science, 1993, 260, 920-926.

14 W. H. Ryu, S. W. Min, K. E. Hammerick, M. Vyakarnam, R. S. Greco, F. B. Prinz and R. J. Fasching, The construction of three-dimensional micro-fluidic scaffolds of biodegradable polymers by solvent vapor based bonding of micro-molded layers, Biomaterials, 2007, 28, 1174-1184.

15 L. D. Zhang and L. J. Hui, Bioengineering: Bile ducts regenerated, Nature, 2017, 547, 171-172.

16 P. Bianco and P. G. Robey, Stem cells in tissue engineering, Nature, 2001, 414, 118-121.

17 T. Z. Li, C. Z. Jin, B. H. Choi, M. S. Kim, Y. J. Kim, S. R. Park, J. H. Yoon and B. H. Min, Using cartilage extracellular matrix (CECM) membrane to enhance the reparability of the bone marrow stimulation technique for articular cartilage defect in canine Model, Adv. Funct. Mater., 2012, 22, 4292-4300.

18 K. H. Choi, B. H. Choi, S. R. Park, B. J. Kim and B. H. Min, The chondrogenic differentiation of mesenchymal stem cells on an extracellular matrix scaffold derived from porcine chondrocytes, Biomaterials, 2010, 31, 5355-5365.

19 C. L. Zhu, S. Pongkitwitoon, J. C. Qiu, S. Thomopoulos and Y. N. Xia, Design and fabrication of a hierarchically structure scaffold for tendon-to-bone repair, Adv. Mater., 2018, 30, 1707306.

20 K. Wang, X. Wang, C. S. Han, W. D. Hou, J. Y. Wang, L. Y. Chen and Y. Luo, From micro to macro: the hierarchical design in a micropatterned scaffold for cell assembling and transplantation, Adv. Mater., 2017, 29, 1604600.

21 Y. Sumita, M. J. Honda, T. Ohara, S. Tsuchiya, H. Sagara, H. Kagami and M. Ueda, Performacne of collagen sponge as a 3-D scaffold for tooth-tissue engineering, Biomaterials, 2006, 27, 3238-3248.

22 P. Feng, J. Y. He, S. P. Peng, C. D. Gao, Z. Y. Zhao, S. X. Xiong and C. J. Shaui, Characterizations and interfacial reinforcement mechanisms of multicomponent biopolymer based scaffold, Mater. Sci. Eng. Carbon, 2019, 100, 809-825.

23 P. Feng, P. Wu, C. D. Gao, Y. W. Yang, W. Guo, W. J. Yang and C. J. Shuai, A multimaterial scaffold with tunable properties: toward bone tissue repair, Adv. Sci., 2018, 5, 1700817.

24 C. D. Gao, S. P. Peng, P. Feng and C. J. Shuai, Bone biomaterials and interactions with stem cells, Bone Res., 2017, 5, 17059.

25 R. S. Tare, F. Khan, G. Tourniaire, S. M. Morgan, M. Bradley and R. O. C. Oreffo, A microarray approach to the identification of polyurethanes for the isolation of human skeletal progenitor cells and augmentation of skeletal cell growth, Biomaterials, 2009, 30, 1045-1055.

26 K. Park, Y. M. Ju, J. S. Son, K. D. Ahn and D. K. Han, Surface modification of biodegradable electrospun nanofiber scaffolds and their interaction with fibroblasts, J. Biomater. Sci., Polym. Ed., 2007, 18, 369-382.

27 H. H. Lu, J. A. Cooper, S. Manuel, J. W. Freeman, M. A. Attawia, F. K. Ko and C. T. Laurencin, Anterior cruciate ligament regeneration using braided biodegradable scaffolds: in vitro optimization studies, Biomaterials, 2005, 26, 4805-4816.

28 R. S. Bhati, D. P. Mukherjee, K. J. McCarthy, S. H. Rogers, D. F. Smith and S. W. Shalaby, The growth of chondrocytes 
into a fibronectin-coated biodegradable scaffold, J. Biomater. Sci., Polym. Ed., 2001, 56, 74-82.

29 F. Mwale, H. T. Wang, V. Nelea, L. Luo, J. Antoniou and M. R. Wertheimer, The effect of glow discharge plasma surface modification of polymers on the osteogenic differentiation of committed human mesenchymal stem cells, Biomaterials, 2006, 27, 2258-2264.

30 Y. Yang, K. Kulangara, R. T. S. Lam, R. Dharmawan and K. W. Leong, Effects of topographical and mechanical property alterations induced by oxygen plasma modification on stem cell behavior, ACS Nano, 2012, 6, 8591-8598.

31 Y. Guo, D. L. Shi, H. S. Cho, Z. Y. Dong, A. Kulkarni, G. M. Pauletti, W. Wang, J. Lian, W. Liu, L. Ren, Q. Q. Zhang, G. K. Liu, C. Huth, L. M. Wang and R. C. Ewing, In vivo imaging and drug storage by quantum-dot-conjugated carbon nanotubes, Adv. Funct. Mater., 2008, 18, 2489-2497.

32 Y. Q. Wan, J. Yang, J. L. Yang, J. Z. Bei and S. G. Wang, Cell adhesion on gaseous plasma modified poly-(L-lactide) surface under shear stress field, Biomaterials, 2003, 24, 3757-3764.

33 H. Shin, S. Jo and A. G. Mikos, Biomimetic materials for tissue engineering, Biomaterials, 2003, 24, 4353-4364.

34 Z. W. Ma, M. Kotaki, R. Inai and S. Ramakrishna, Potential of nanofiber matrix as tissue-engineering scaffolds, Tissue Eng., 2005, 11, 101-109.

35 T. Desmet, R. Morent, N. De Geyter, C. Leys, E. Schacht and P. Dubruel, Nonthermal plasma technology as a versatile strategy for polymeric biomaterials surface modification: a review, Biomacromolecules, 2009, 10, 2351-2378.

36 E. A. Ryan, J. R. T. Lakey, B. W. Paty, S. Imes, G. S. Korbutt, N. M. Kneteman, D. Bigam, R. V. Rajotte and A. M. J. Shapiro, Successful islet transplantation continued insulin reserve provides long-term glycemic control, Diabetes, 2002, 51, 2148-2157.

37 R. P. Robertson, Medical progress: islet transplantation as a treatment for diabetes - a work in progress, N. Engl. J. Med., 2004, 350, 694-705.
38 P. Fiorina, A. M. J. Shapiro, C. Ricordi and A. Secchi, The clinical impact of islet transplantation, Am. J. Transplant., 2008, 8, 1990-1997.

39 L. P. Rutzky, S. Bilinski, M. Kloc, T. Phan, H. M. Zhang, S. M. Katz and S. M. Stepkowski, Microgravity culture condition reduces immunogenicity and improves function of pancreatic islets1, Transplantation, 2002, 74, 13-21.

40 J. Park, B. Andrade, Y. Seo, M. J. Kim, S. C. Zimmerman and H. Kong, Engineering the surface of therapeutic "living" cells, Chem. Rev., 2018, 118, 1664-1690.

41 P. Orning, K. S. Hoem, A. E. Coron, G. Skjak-Braek, T. E. Mollnes, O. L. Brekke, T. Espevik and A. M. Rokstad, Alginate microsphere compositions dictate different mechanisms of complement activation with consequences for cytokine release and leukocyte activation, J. Controlled Release, 2015, 229, 58-69.

42 K. E. Crompton, J. D. Goud, R. V. Bellamkonda, T. R. Gengenbach, D. I. Finkelstein, M. K. Horne and J. S. Forsythe, Polylysine-functionalised thermoresponsive chitosan hydrogel for neural tissue engineering, Biomaterials, 2007, 28, 441-449.

43 A. Hokugo, T. Takamoto and Y. Tabata, Preparation of hybrid scaffold from fibrin and biodegradable polymer fiber, Biomaterials, 2006, 27, 61-67.

44 W. J. Li, J. A. Cooper, R. L. Mauck and R. S. Tuan, Fabrication and characterization of six electrospun poly(alpha-hydroxy ester)-based fibrous scaffolds for tissue engineering applications, Acta Biomater., 2006, 2, 377-385.

45 Y. Luo and M. S. Shoichet, A photolabile hydrogel for guided three-dimensional cell growth and migration, Nat. Mater., 2004, 3, 249-253.

46 I. Kreiser-Saunders and H. R. Kricheldorf, Polyactones, 39 $\mathrm{Zn}$ lactate-catalyzed copolymerization of L-lactide with glycolide or epsilon-caprolactone, Macromol. Chem. Phys., 1998, 199, 1081-1087.

47 H. J. Sung, C. Meredith, C. Johnson and Z. S. Galis, The effect of scaffold degradation rate on three-dimensional cell growth and angiogenesis, Biomaterials, 2004, 25, 5735-5742. 